\title{
Performance of High-Permittivity Ceramic-Polymer Composite as a Substrate for UHF RFID Tag Antennas
}

\author{
A. A. Babar, ${ }^{1}$ V. A. Bhagavati, ${ }^{2}$ L. Ukkonen, ${ }^{1}$ A. Z. Elsherbeni, ${ }^{3}$ P. Kallio, ${ }^{2}$ and L. Sydänheimo' \\ ${ }^{1}$ Rauma Research Unit, Department of Electronics, Tampere University of Technology, 26100 Rauma, Finland \\ ${ }^{2}$ Micro and Nano Systems Research Group, Department of Automation Science and Engineering, \\ Tampere University of Technology, 33720 Tampere, Finland \\ ${ }^{3}$ Electrical Engineering Department, The University of Mississippi, University, MS 38677, USA \\ Correspondence should be addressed to A. A. Babar, abdul.babar@tut.fi \\ Received 12 December 2011; Accepted 4 April 2012 \\ Academic Editor: Carles Fernández-Prades
}

Copyright (๑) 2012 A. A. Babar et al. This is an open access article distributed under the Creative Commons Attribution License, which permits unrestricted use, distribution, and reproduction in any medium, provided the original work is properly cited.

A high-permittivity ceramic-polymer composite substrate is fabricated and proposed for its potential use in UHF RFID tags. The substrate is developed using high-permittivity Barium Titanate $\left(\mathrm{BaTiO}_{3}\right)$ ceramic powder mixed with polydimethylsiloxane (PDMS) polymer. The resulting composite achieves a soft, hydrophobic, heat resistant, low loss, and flexible material with high dielectric constant. The percentage of the ceramic powder in the composite helps in achieving variable permittivity values. When this material is used as a substrate for a tag antenna, it will help the tag to be reduced in size, to conform to uneven or rough surface, and to be less vulnerable to breakage or other environmental damages. A small passive UHF RFID tag antenna is designed, fabricated, and attached to this type of composite substrate, to demonstrate the performance of this composite material.

\section{Introduction}

There have been a growing demand and progress in the field of wireless communication and identification in recent years. The use of Radio frequency identification (RFID) technology as an effective and reliable way of tracking and sensing various objects is gaining much importance recently. An RFID system is composed of two main components, the reader and the tag. A commonly used passive RFID tag is composed of an IC chip and an antenna, which contains its own unique identification code (ID) [1]. The identification code is sent back to the reader when the tag is interrogated or energized through backscattered modulation of the incident continuous wave [2]. The input impedance of a passive UHF RFID tag antenna and reflectivity rely on the physical properties of the substrate or material it is attached to and on the antenna's geometry. The permittivity of the substrate has a great impact on the size of the tag antenna. Using higherpermittivity substrate can be useful in reducing the size of the tag antenna.

Substrates play a vital role in the development and use of electronic devices. Flexible substrates have gained much importance in recent years. Different techniques and types of materials have been used to develop flexible electronic substrates [3-6]. One of the most commonly used flexible plastic substrates is polyester, PEEK (polyaryletheretherketone) and polyimides $[7,8]$. Several techniques are being studied and used to embed and integrate electronic components with these flexible substrate materials $[9,10]$. There has been a growing interest in the development of flexible antennas using flexible substrates. This gives the advantage of having conformal antennas being placed and attached to uneven shaped structures and surfaces. It is more desirable to use flexible substrate materials that are less vulnerable to heat, water, and other damaging effects.

The size of the RFID tag antenna is also one of the major concerns for RFID applications. Reducing the size of the tag antenna comes at the cost of its performance, such as gain and efficiency of the antenna. Therefore, achieving a small RFID tag antenna, with improved efficiency is an important and challenging task for antenna designers. Using highpermittivity substrates is considered for reducing the overall size and for having compact antenna structures. Several commercially available substrates, with high permittivity values, 
have hard and rigid structures. They are more breakable, less conformal, and not suitable for uneven surfaces.

In this paper, new high-permittivity substrates have been developed and tested for RFID tag antenna designs. The substrates are soft and flexible in nature, resistant to water, heat, breaking, and other damaging effects. The substrates have been fabricated by mixing a ceramic powder known as barium titanate $\left(\mathrm{BaTiO}_{3}\right)$ with polydimethylsiloxane (PDMS) polymer. Barium titanate is a ferroelectric material, with high dielectric constant values. The dielectric properties of the substrates depend on the fabrication process, the ratio and the type of ceramic powder used in the composite. The permittivity value of the substrates can be changed by changing the percentage of ceramic powder in PDMS. This provides the flexibility to achieve desired high permittivity values of the substrates.

To demonstrate the performance of the composite substrate for an RFID tag, a small passive UHF RFID tag antenna is designed and fabricated on a thin sheet of FR4. It is then attached to the ceramic-polymer substrate. This was useful to accurately fabricate the small tag antenna and analyze the performance of the substrate. The antenna is designed using miniaturization techniques to reduce the size of the tag antenna and make it more compact and suitable for various objects. The substrate of the antenna makes it possible to place and attach the tag on irregular surfaces and rough terrains. This type of high permittivity composite substrate allows the possibility of achieving small flexible thin tags for future applications which may include the design of fully flexible on-metal, thin, conformal, and embedded tags using inkjet printing.

The rest of the paper is organized as follows. In Section 2, properties of the ceramic and polymer will be discussed. This will be followed by the fabrication process of the composite substrate in Section 3. Section 4 will focus on the design and development of the tag antenna. Simulations and measurements results are discussed and shown in Section 5, followed by the conclusions in Section 6 .

\section{Properties of Ceramic and Polymer}

In this section, the properties and characteristics of both the ceramic powder and the polymer used in this experiment will be discussed. The ceramic powder used in the composite substrate is a ferroelectric material known as barium titanate $\left(\mathrm{BaTiO}_{3}\right)$. Polydimethylsiloxane (PDMS) polymer is mixed with the ceramic powder and fabricated, to make it a soft and flexible solid composite substrate.

2.1. Characteristics of PDMS. PDMS is most widely used type of a silicon-based organic polymer, belonging to a group of siloxanes. It is available in fluid, elastomer, and resin form [11]. The chemical structure of PDMS is stated in [11].

PDMS materials have high thermal stability, low surface tension, characteristic pressure and bulk viscosity. They are transparent, hydrophobic in nature and durable at high temperatures. Other PDMS properties include lubricity, shear stability, high dielectric strength, antifriction, and good resistance to UV radiations [11, 12].

In the elastomer form, PDMS is suitable for flexible substrates. Elastomeric PDMS structures are typically fabricated using viscous liquid and a liquid cross-linking agent. Among other factors such as curing time and temperature, the bending stiffness depends on the amount of cross-linking agent used. Larger amounts of PDMS result in more rigid structures [12].

The most common applications of PDMS include rubber molds, surfactants, water repellents, adhesives, personal care and cosmetics, dielectric encapsulation, hydraulic fluids, lubricants, fuser oil, and protectants. It can also be used in various other applications such as contact lenses, medical and microfluidic devices, and applications [11-14].

2.2. Characteristics of $\mathrm{BaTiO}_{3}$. Barium titanate $\left(\mathrm{BaTiO}_{3}\right)$ is a ferroelectric ceramic powder, with piezoelectric properties [15]. It is insoluble in water and alkalis, whereas soluble in some acids such as sulfuric, hydrofluoric, and hydrochloric acids. It usually has very high permittivity values. It can acquire four different shapes of crystals, cubic, tetragonal, orthorhombic, and rhombohedral. However, the permittivity depends on many factors, such as the grain size, shape and size of the crystals, impurities, and on processing techniques [16]. Ferroelectric ceramics can be used in several applications, such as capacitors, thermostatic devices, light switches, current controllers and to generate ultrasonic energy [15]. Similarly, barium titanate is used to manufacture many electronic components, such as multilayer capacitors [17], positive temperature coefficient (PTC) thermistors, transducers, and tunable phase shifters $[18,19]$.

Due to recent advances in electronics, much attention has been focused on small and miniaturized electronic devices. Growing interest is targeting the use of high permittivity material in electronics industry to decrease the size of electronic and wireless components and devices.

\section{Fabrication Technique Used for Ceramic-Polymer Composite Substrate Material}

In this section, design and fabrication technique of the composite substrate is discussed. A stepwise approach is used to explain the fabrication process of $\mathrm{PDMS}_{-}-\mathrm{BaTiO}_{3}$ (barium titanate) composite substrate. A similar approach has also been used in [20]. The PDMS used is Sylgard-184, which is supplied as a two component Kit containing a "base prepolymer" (the viscous liquid) and a "curing agent" (the liquid cross-linking agent) manufactured by Dow Corning [21].

Following are the steps to make the ceramic-polymer composite substrate.

(1) First, the base prepolymer is mixed with the curing agent in a mass ratio of 10 (pre-polymer): 1 (curing agent). The mixture is then mixed vigorously for $5-10$ minutes in a plastic container, which enables uniform cross-linking. 


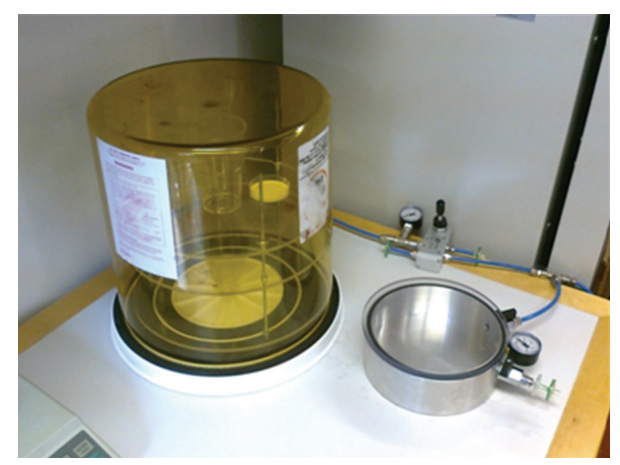

FIGURE 1: Degassing chambers used to degas the samples.

(2) After mixing PDMS (the base pre-polymer with the curing agent), it is left for degassing in a degassing chamber for 15-20 minutes. This helps in extracting possible air bubbles formed during the process of mixing. The degassing chambers used for degassing of the samples are shown in Figure 1. In this step, the samples are placed in the bigger degassing chamber, to accommodate the plastic glass used for mixing.

(3) The degassed mixture of PDMS is mixed with $\mathrm{BaTiO}_{3}$ ceramic powder. The ceramic powder is provided from Sachtleben Pigments [22]. The percentage of ceramic powder mixed with PDMS is on a volumebased ratio, as defined in (1) [20]:

$$
\nu \%=\frac{C_{m} / C_{\rho}}{C_{m} / C_{\rho}+P_{m} / P_{\rho}} \times 100 \% .
$$

In the previous equation $C_{m}$ and $C_{\rho}$ refer to the mass and density of the ceramic $\left(\mathrm{BaTiO}_{3}\right)$, respectively, whereas, $P_{m}$ and $P_{\rho}$ are the mass and density of the polymer (PDMS).

In this experiment, $15 \%$ and $25 \%$ of $\mathrm{BaTiO}_{3}$ ceramic powder are mixed with PDMS. The $\mathrm{BaTiO}_{3}$ ceramic powder provided by Sachtleben Pigments has a grain size equal to $80 \mathrm{~nm}$. The density of $\mathrm{BaTiO}_{3}$ is equal to $6.02 \mathrm{~g} / \mathrm{cm}^{3}$, whereas the density of PDMS is equal to $1.05 \mathrm{gm} / \mathrm{cm}^{3}$.

(4) After adding PDMS with the ceramic powder, vigorous stirring is done for about 15-20 min, as shown in Figure 2. The mixture is stirred until it transforms into a smooth paste. A smooth white paste without any lumps is a good indicator of a good mixture.

(5) The mixture is then poured into a small Petri dish on a flat surface, to have an even distribution of the thick paste inside the container. This mixture can be poured into different types of molds, depending on the desired shape, as shown in Figure 3.

(6) After pouring the mixture into the Petri dish, it is again left for degassing for a continuous time period of 5-6 hours, to remove excessive air bubbles. The first 5-6 hours are considered crucial, as they help in removing the maximum amount of air bubbles. It has been observed that, even after 5-6 hours of degassing,

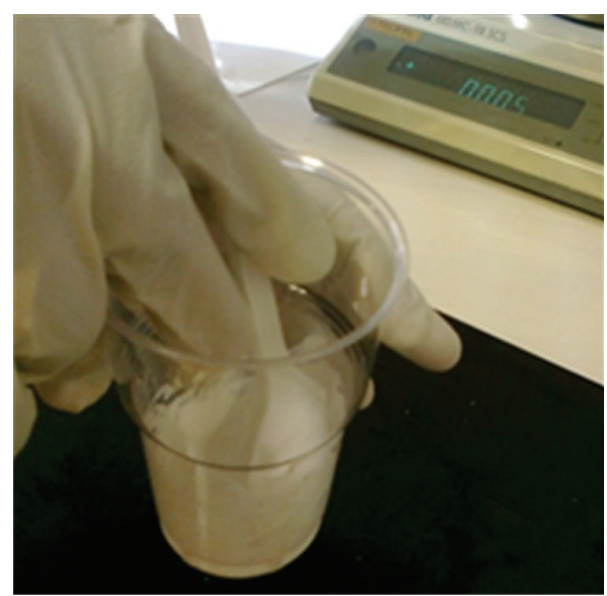

FIGURE 2: Vigorous mixing of PDMS ceramic powder with PDMS mixture.

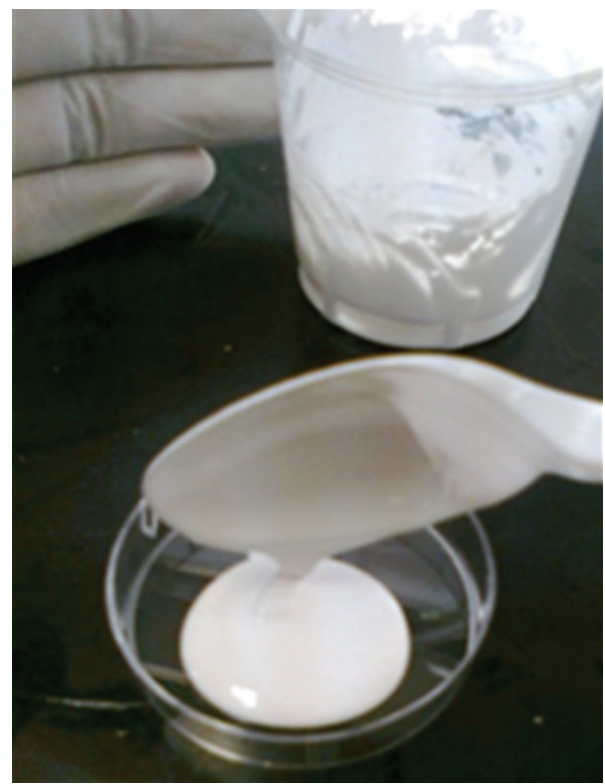

Figure 3: Pouring the well-mixed paste into a Petri dish.

few air bubbles are still popping out of the mixture. Therefore, to ensure that the mixture has no air bubbles left, it is kept under vacuum pressure for 30 hours, as shown in Figure 4. Usually a solid structure is achieved within 24 hours of curing. This process can be made faster, by heating the mixture after degassing. However, the heating of the composite can make the substrate structure more rigid, depending on the applied temperature.

(7) After curing, the composite substrate is carefully taken out of the Petri dish.

As shown in Figure 5, the final composite substrate is soft and flexible with a smooth surface. The substrate can be softer and more flexible by changing the thickness of the substrate. 


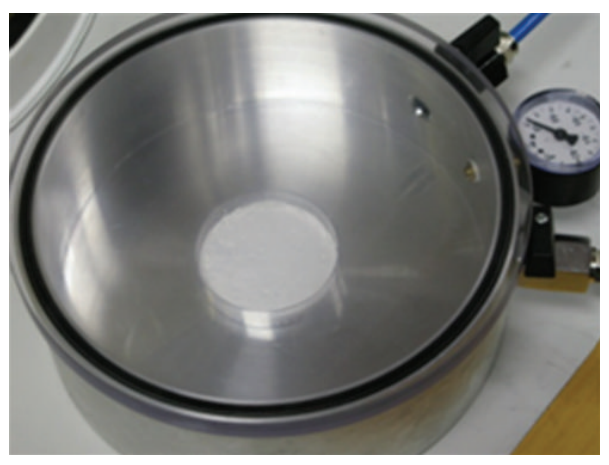

(a)

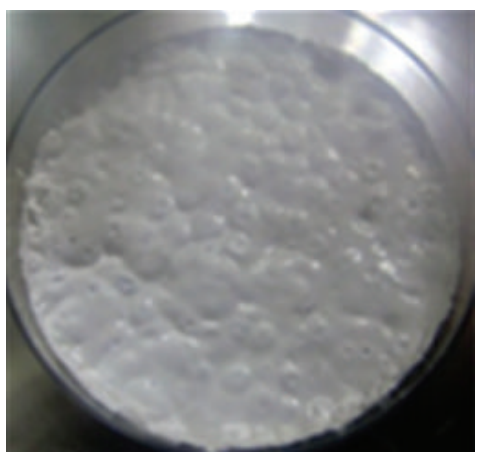

(b)

Figure 4: (a) Small degassing chamber, to degas the final composite mixture in room temperature. (b) Extraction of air bubbles from the surface of the composite mixture during degassing.

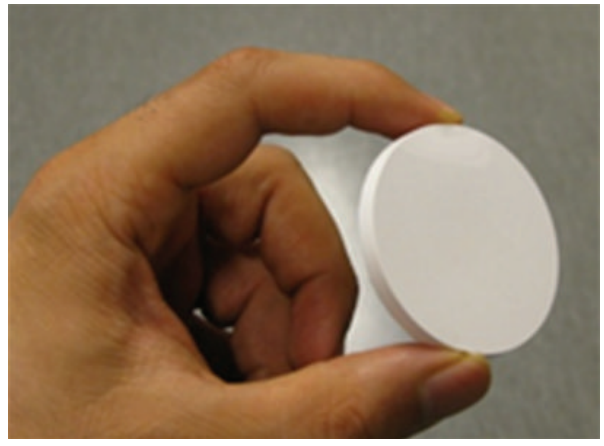

(a)

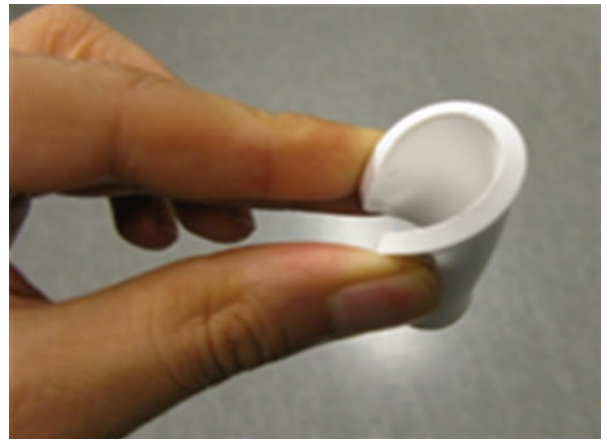

(b)

FIGURE 5: Final solid shape of the flexible composite substrate.

\section{Antenna Design Technique}

In this section, UHF RFID tag antenna design techniques are discussed. The tag antenna is designed using Ansoft HFSS v.12 [23]. A multilayer substrate approach is used to design and fabricate the tag antenna. The tag antenna is first fabricated on a thin sheet of FR4 substrate layer of $0.16 \mathrm{~mm}$ thickness, using milling machine. Later the tag is attached to the ceramic-polymer composite substrate, as stated in Section 3.

Alien Higgs $3 \mathrm{IC}$ with $-18 \mathrm{dBm}$ sensitivity is used in the tag antenna [24]. The IC provided by the manufacture comes with a strap attachment. In order to take the effect of the strap into account, $0.2 \mathrm{pF}$ of parasitic capacitance is added in parallel to the nominal IC impedance. The tag is matched at $860 \mathrm{MHz}$.

Figure 6 shows the tag antenna in a form of a round symmetric dipole structure. The IC strap of the tag is attached between the two feeding pads with dimensions represented by $a$ and $b$. Line $d$ connects the two feeding pads with the two symmetric halves of the dipole structure along the $x$-axis, as shown in Figure 6. The two halves of the dipole structure are shorted or T-matched along the $x$-axis using line " $g$ ". The edge " $n$ " of the two sides of the dipole structure is kept at a distance of $1 \mathrm{~mm}$ from each side. Table 1 lists the dimensions of the geometrical parameters of the tag, shown in Figure 6.

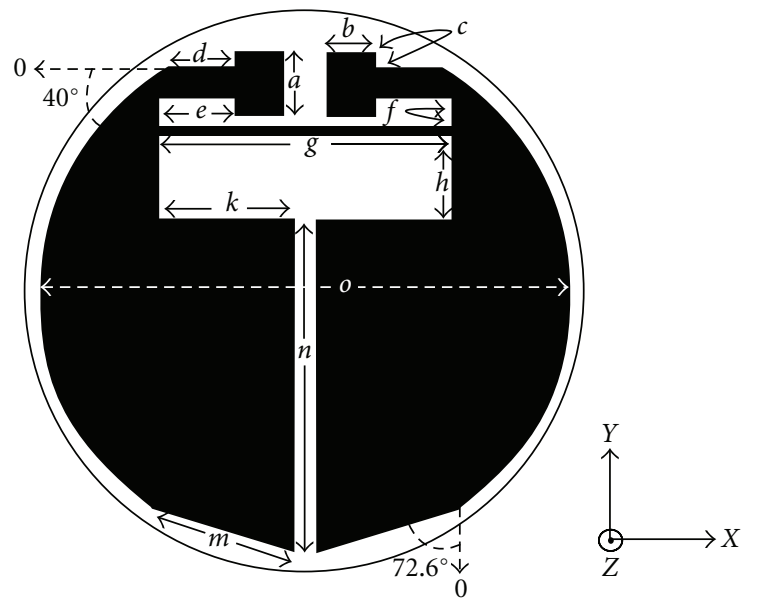

FIGURE 6: Geometrical parameters of the designed tag antenna.

Miniaturization techniques are used to reduce the overall size of the tag. T-matching technique is useful in reducing the overall size of the tag, by increasing the inductance of the antenna. T-matching works as an impedance transformer [25]. The length and width of the shorted line are of considerable importance in tuning the antenna. The tuning 
TABLE 1: Geometrical parameters of the tag.

\begin{tabular}{lcc}
\hline Line & Length $(\mathrm{mm})$ & Width $(\mathrm{mm})$ \\
\hline$a$ & 3.25 & - \\
$b$ & 2.5 & - \\
$c$ & 0.75 & - \\
$d$ & 3.55 & 2 \\
$e$ & 3.75 & 2 \\
$f$ & 1 & - \\
$g$ & 14.5 & 0.5 \\
$h$ & 4.15 & - \\
$k$ & 6.75 & - \\
$m$ & 7.5 & - \\
$n$ & 16.6 & - \\
$o$ & 26.5 & - \\
\hline
\end{tabular}

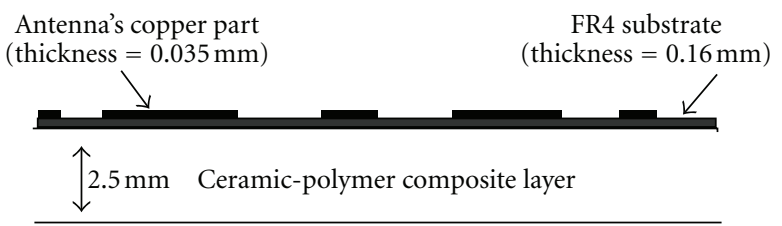

FIgURE 7: Side view of the tag antenna substrate.

and matching of the tag antenna to the desired frequency can also be done by changing the distance " $f$ " of the shorted line " $g$ " from the feeding pads.

A round-shaped tag with a feeding point aligned in the $x$-axis of the tag antenna provides a longer current path. This helps in reducing the overall size of the tag antenna and in achieving a compact shape of the tag. The tag antenna can be tuned further by increasing or decreasing the gap between the two " $n$ " edges or by adjusting the separation distance between the two halves of the tag. Figure 7 shows the side view of the tag, demonstrating a multilayer structure of the tag's substrate.

Figure 7 illustrates two main layers of the substrate. First the tag is fabricated on a thin sheet of FR4, with $35 \mu \mathrm{m}$ copper cladding, using milling machine. This is attached to the custom made composite substrate layer of $2.5 \mathrm{~mm}$ thickness. The two layers were attached using commercially available adhesive glue. The effect of the glue is considered negligibly small to affect the tag antennas performance. The two layers are attached carefully, to avoid any air gap in between, as it can alter the effective permittivity of the substrate. The relative permittivity value of the ceramic-polymer composite layer in this experiment is approximately equal to 10.5 and 8 for $25 \%$ and $15 \%$ of $\mathrm{BaTiO}_{3}$ mixtures, respectively. This is measured by agilent dielectric probe and verified by the experimental results presented in $[26,27]$.

\section{Simulation and Measurement Results}

This section focuses on the simulation and measurement results of the tag, on a multilayer substrate. Section 5.1

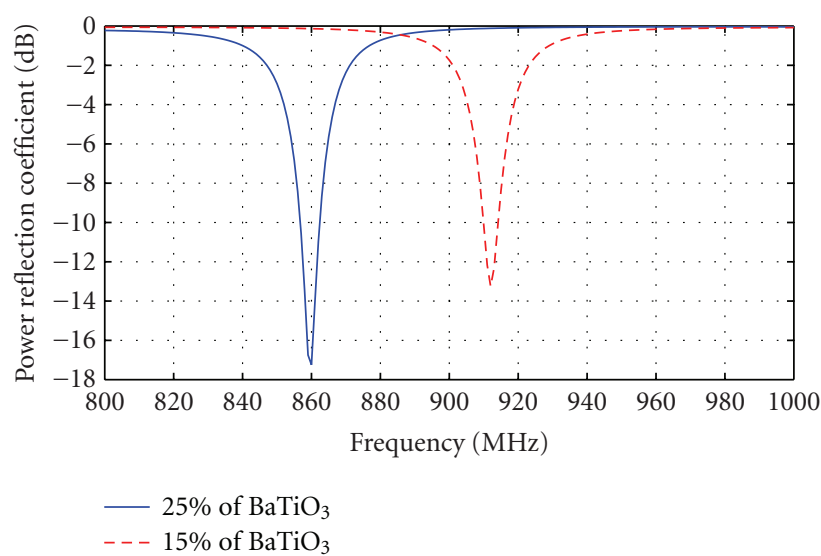

FIGURE 8: Simulated power reflection coefficient of the RFID tag antenna with $25 \%$ and $15 \%$ of $\mathrm{BaTiO}_{3}$ concentration in the composite substrate material.

demonstrates the simulated power reflection coefficient and conjugate impedance matching of the designed tag antenna. In Section 5.2, measured results of the tag are discussed.

5.1. Simulation Results of the Tag Antenna. The transfer of power between the complex load (IC) impedance and the complex source (tag antenna) impedance is analyzed using (2). The power reflection coefficient can be analyzed by considering the ratio of the incident power on the tag antenna $\left(P_{\mathrm{tag}}\right)$ and the power reflected back $\left(P_{\mathrm{rfl}}\right)$, as defined in $[28,29]$ such that

$$
\frac{P_{\mathrm{rfl}}}{P_{\mathrm{tag}}}=\left|\frac{Z_{\mathrm{IC}}-Z_{a}^{*}}{Z_{\mathrm{IC}}+Z_{a}}\right|^{2}
$$

In the previous equation $Z_{a}=R_{a}+j X_{a}$ is the impedance of the tag antenna, whereas $Z_{\mathrm{IC}}=R_{\mathrm{IC}}+j X_{\mathrm{IC}}$ is the impedance of the chip. The superscript $\left(^{*}\right)$ denotes the complex conjugate. Lower value of the power reflection coefficient at the desired operating frequency indicates an optimal power transfer. An optimal power transfer enables the maximum achievable realized gain and read range of the tag.

The simulated power reflection coefficient of the tag is shown in Figure 8. The tag is matched to $860 \mathrm{MHz}$ with a substrate having $25 \%$ of $\mathrm{BaTiO}_{3}$ and to $915 \mathrm{MHz}$ with $15 \%$ of $\mathrm{BaTiO}_{3}$ composite substrate.

Figure 9 shows the input impedance of the tag antenna and the chip, on top of a $25 \%$ ceramic composite substrate, illustrating a good conjugate matching of the tag antenna with the chip, at $860 \mathrm{MHz}$. This is essential to minimize the reflection loss at this junction and improve the power transmission and maximize the read range.

5.2. Measurement Results of the Tag Antenna. The tag antenna is measured using Tagformance RFID measurements device in an anechoic chamber by Voyantic Ltd., [30]. The anechoic chamber is designed to measure the UHF RFID tag antennas. The chamber consists of a rotating table and a linearly polarized reader antenna. The linearly polarized 


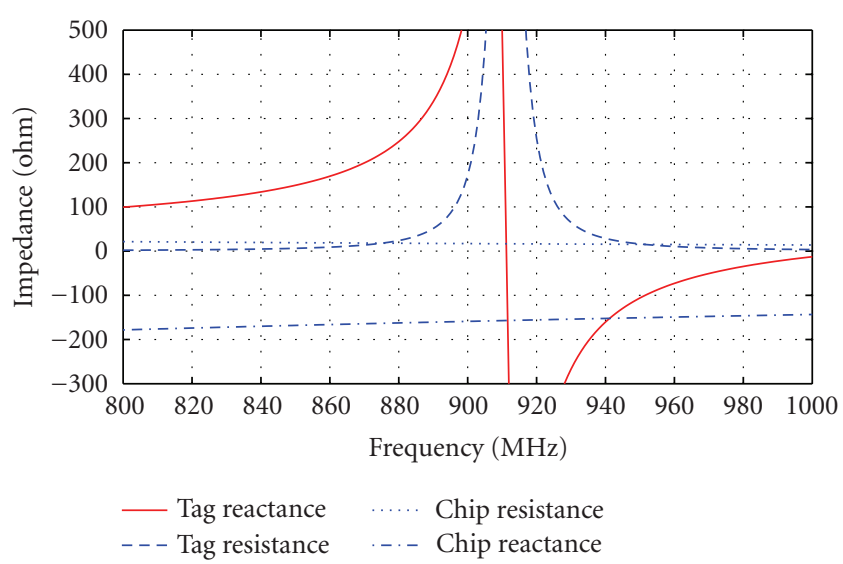

FIGURE 9: Simulated chip and antenna impedance of the UHF RFID tag on top of a $25 \%$ ceramic-polymer composite.

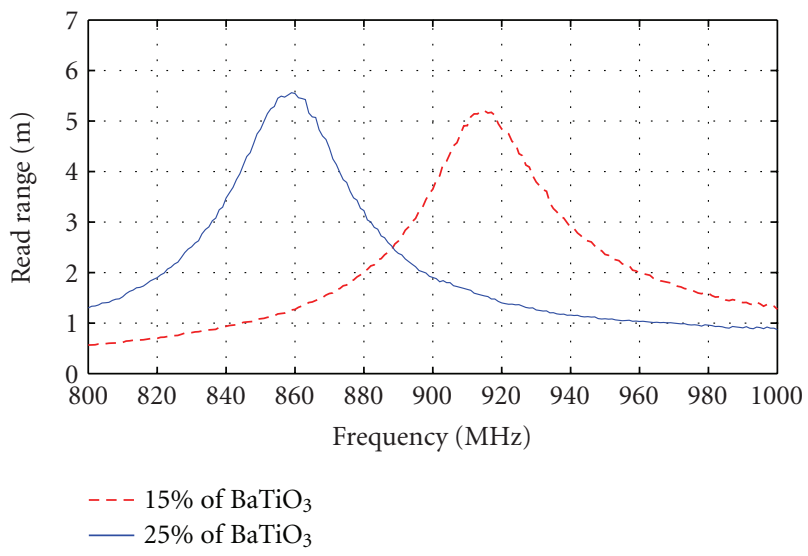

Figure 10: Calculated read range $\left[d_{\mathrm{tag}}\right]$ in $+z$ direction of the tag antenna, with $15 \%$ and $25 \%$ of $\mathrm{BaTiO}_{3}$ concentration in the composite substrates, based on measured results and (3).

reader antenna is connected to the Tagformance measurement equipment.

Read range of the tag is calculated using the measured results from the Tagformance, using [31].

$$
d_{\mathrm{tag}}=\frac{\lambda}{4 \pi} \sqrt{\frac{1.64 P_{\mathrm{ERP}}}{L_{\mathrm{fwd}} P_{\mathrm{th}}}} .
$$

In (3), " $d_{\mathrm{tag}}$ " represents the read range of the tag antenna. " $L_{\mathrm{fwd}}$ " is the measured path loss from the generator's output port to the input port of a hypothetic isotropic antenna placed at the tag's location. The forward path loss is achieved from the measured calibration data obtained from Tagformance measurement device. The European effective radiated power " $P_{\mathrm{ERP}}$ " is considered to be equal to $2 \mathrm{~W}(33 \mathrm{dBm})$, as stated in [32]. " $P_{\text {th }}$ " represents the measured threshold power in the forward direction, from the transmitter to the tag antenna. The threshold power is the minimum continuous wave power from the transmitter, required to turn on the IC and enable the tag to send a response to EPC Gen 2 protocol's query command. The read range of the tag antenna with $15 \%$

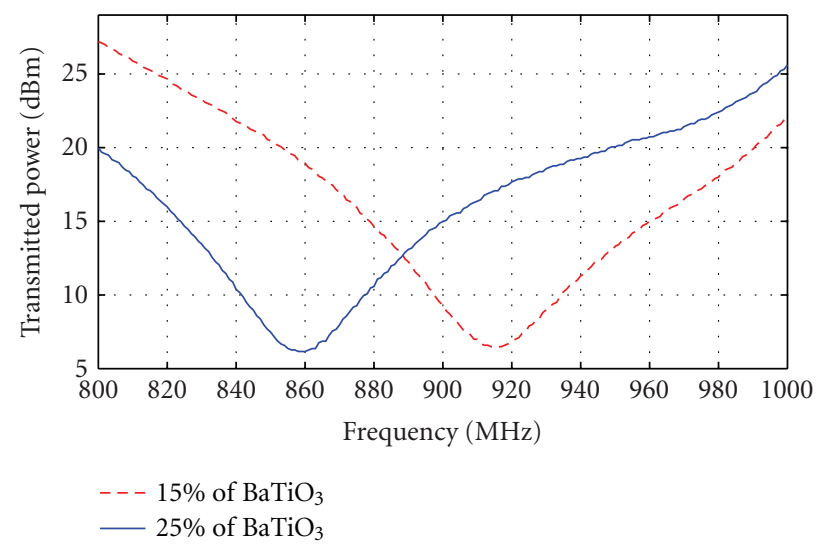

FIGURE 11: Measured threshold transmitted power in $+z$ direction of the tag antenna, with $15 \%$ and $25 \%$ of $\mathrm{BaTiO}_{3}$ concentration in the composite substrates.

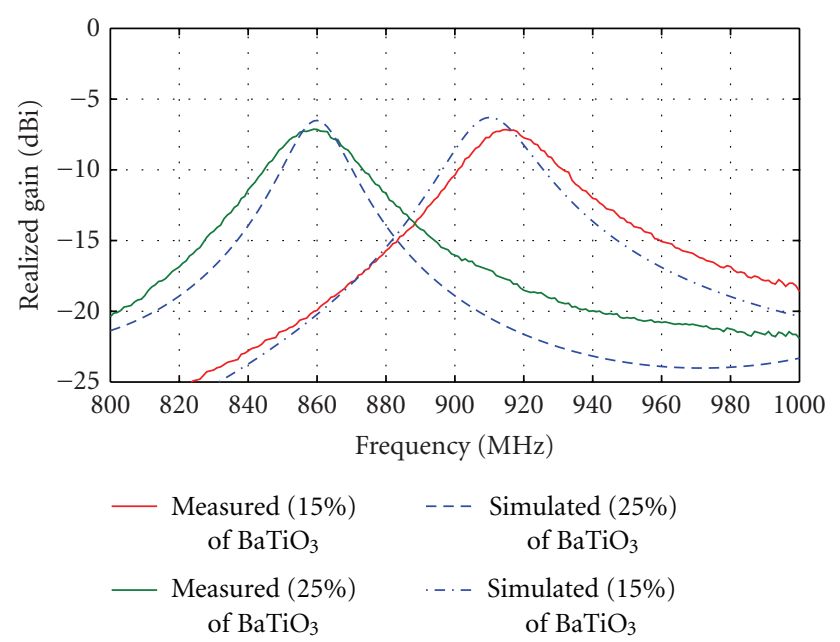

FIGURE 12: Simulated and measured realized gain in " $+z$ " direction of the tag antenna with $15 \%$ and $25 \%$ of $\mathrm{BaTiO}_{3}$ concentration in the composite substrates.

and $25 \%$ of $\mathrm{BaTiO}_{3}$ composite substrates, calculated using the measured results from (3), is shown in Figure 10.

According to Figure 10, the maximum read range achieved is between 5 and 6 meters at $860 \mathrm{MHz}$ and $915 \mathrm{MHz}$. Figure 11 shows the threshold power of the tag required to turn on the IC, with $15 \%$ and $25 \% \mathrm{BaTiO}_{3}$ composite substrates.

The measured realized gain of the tag antenna is analyzed using the path loss measurement data from the Tagformance measuring equipment. This can be described as [31]

$$
G_{r}=\frac{P_{\mathrm{IC}}}{L_{\mathrm{fwd}} \cdot P_{\mathrm{th}}} .
$$

In the previous equation " $P_{\mathrm{IC}}$ " refers to the sensitivity of the IC. " $L_{\mathrm{fwd}}$ " is the forward path loss from the transmitter to the tag antenna, and " $P_{\mathrm{th}}$ " represents the threshold power, as defined in (3). The maximum simulated and measured realized gain of the tag antenna with $15 \%$ and $25 \% \mathrm{BaTiO}_{3}$ composite substrate is shown in Figure 12. 


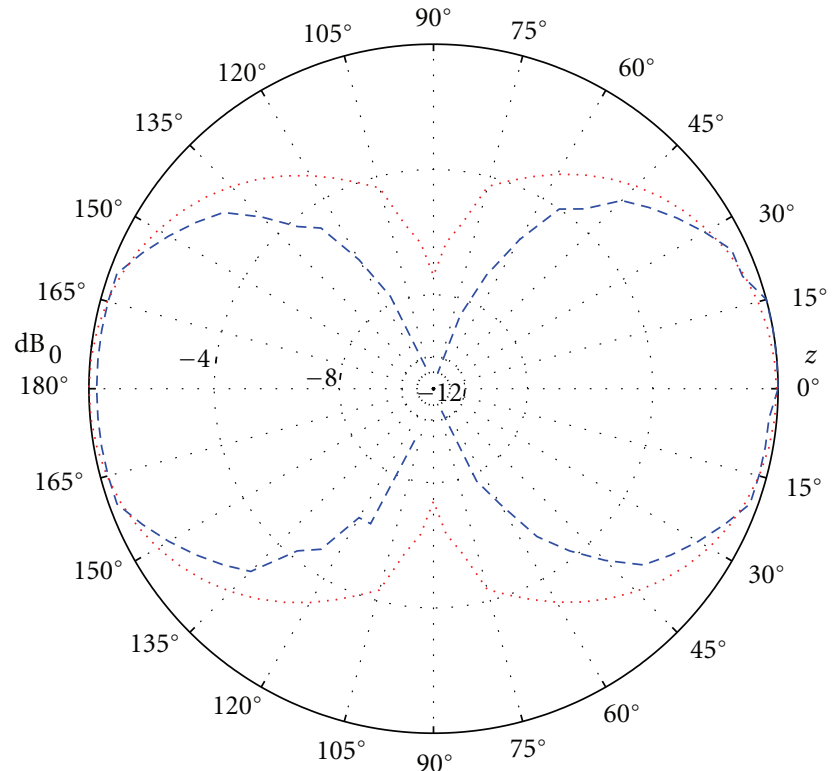

- - - Measured

Simulated

FIGURE 13: Measured and simulated E-plane ( $y z$-plane) of the tags radiation pattern, using normalized gain $(\mathrm{dB})$ values, at $860 \mathrm{MHz}$.

In Figure 12, the measured and simulated realized gains at $860 \mathrm{MHz}$ and $915 \mathrm{MHz}$ show a good agreement with each other. The maximum realized gain achieved at $860 \mathrm{MHz}$ and $915 \mathrm{MHz}$ frequency bands, shows a reasonable performance of such a small tag antenna. Figures 13 and 14 show the simulated and measured normalized gain radiation patterns of the tag at $860 \mathrm{MHz}$.

In Figures 13 and 14, $E$ and $H$ planes of the normalized gain radiation pattern are shown. The measured and simulated radiation patterns show dipole like omnidirectional radiation pattern.

\section{Conclusions}

A ceramic-polymer composite substrate is fabricated and studied for use in UHF RFID tag antennas application. To achieve such a high dielectric substrate, ceramic $\left(\mathrm{BaTiO}_{3}\right)$ powder is fabricated with a polymer known as polydimethylsiloxane. The composite achieves a high permittivity and low loss substrate material. The low-loss nature of the substrate enhances the realized gain and read range of the tag antenna. Various permittivity values can be achieved by changing the percentage of the ceramic in the substrate. To demonstrate this phenomenon, two substrates with $15 \%$ and $25 \%$ of $\mathrm{BaTiO}_{3}$ ceramic were fabricated with the polymer. The composite substrate materials are flexible, low loss, hydrophobic in nature, and heat resistant. This gives the advantage to the tag to conform to several uneven surfaces, without bearing the risk of breaking and possible environmental damages. To demonstrate the performance of the two substrates, a small UHF RFID tag antenna is designed and fabricated

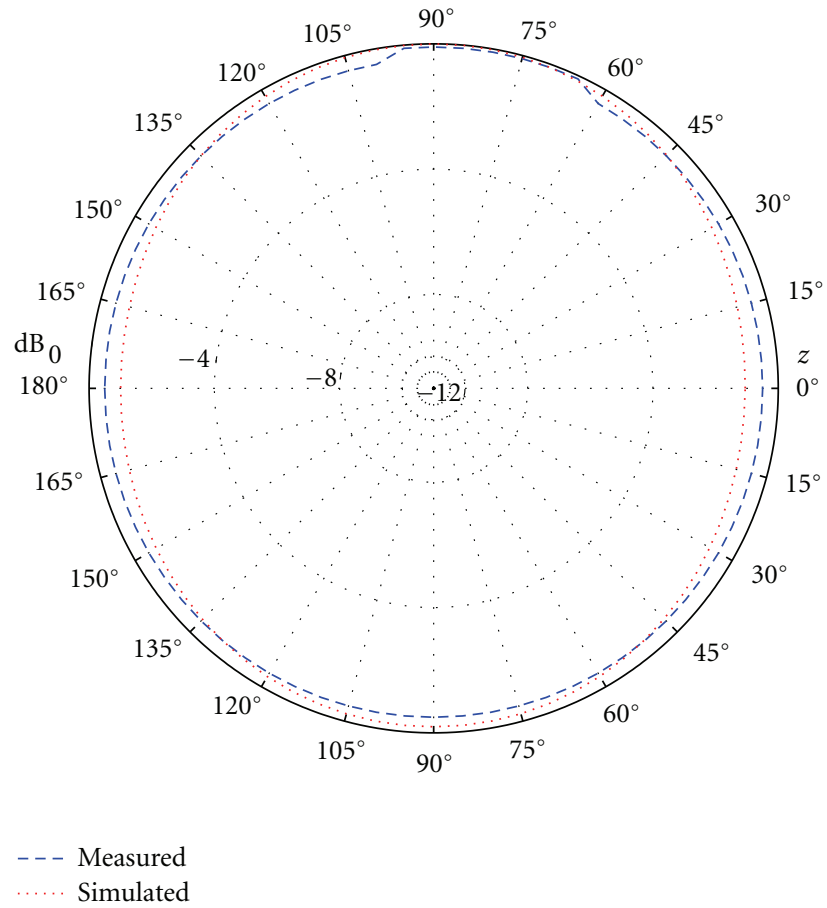

FIGURE 14: Measured and simulated $H$-plane ( $x z$-plane) of the tags radiation pattern, using normalized gain $(\mathrm{dB})$ values, at $860 \mathrm{MHz}$.

on top of a thin FR4 sheet and then glued to the custom made substrate. The measured and simulated results show that the tag antenna has a good performance with respect to its size. This type of small tag antennas can be useful in several applications, due to its small size with respect to its read range and omnidirectional radiation pattern properties. Possible future work may include fabricating thin high permittivity flexible substrates for embedded and on metal tags. To make the thin low-loss substrates fully flexible in nature, the tag antennas can also be printed directly on top of the composite substrate using inkjet printer.

\section{Acknowledgments}

The authors would like to thank Jani Kallioinen and Sachtleben Ltd. for providing $\mathrm{BaTiO}_{3}$ powder samples. The authors would also like to thank Samu Hemmila from Tampere University of Technology for suggestions using PDMS.

\section{References}

[1] D. M. Dobkin, The RF in RFID: passive UHF RFID in Practice, Elsevier, Amsterdam, The Netherlands, 2007.

[2] G. Marrocco and F. Amato, "Self-sensing passive RFID: from theory to tag design and experimentation," in Proceedings of the 39th European Microwave Conference: Science, Progress and Quality at Radiofrequencies (EuMC '09), pp. 1-4, Rome, Italy, October 2009.

[3] A. Sazonov, D. Striakhilev, C. H. O. Lee, and A. Nathan, "Lowtemperature materials and thin film transistors for flexible 
electronics," Proceedings of the IEEE, vol. 93, no. 8, pp. 1420$1428,2005$.

[4] T. H. Sterns, Flexible Printed Circuitry, McGraw-Hill, New York, NY, USA, 1996.

[5] G. P. Crawford, Flexible Flat Panel Displays, John Wiley \& Sons, Chichester, UK, 2005.

[6] K. J. Allen, "Reel to real: prospects for flexible displays," Proceedings of the IEEE, vol. 93, no. 8, pp. 1394-1399, 2005.

[7] S. R. Forrest, "The path to ubiquitous and low-cost organic electronic appliances on plastic," Nature, vol. 428, no. 6986, pp. 911-918, 2004.

[8] K. Jain, M. Klosner, M. Zemel, and S. Raghunandan, "Flexible electronics and displays: high-resolution, roll-to-roll, projection lithography and photoablation processing technologies for high-throughput production," Proceedings of the IEEE, vol. 93, no. 8, pp. 1500-1510, 2005.

[9] K. W. Paik, S. Cho, and J. G. Hyun, "Novel epoxy/BaTiO 3 composite embedded capacitor films embedded in organic substrates," in Proceedings of the International IEEE Conference on Asian Green Electronics (AGEC '04), pp. 68-73, January 2004.

[10] C. H. Wang and G. B. Lee, "Automatic bio-sampling chips integrated with micro-pumps and micro-valves for disease detection," Biosensors and Bioelectronics, vol. 21, no. 3, pp. 419425, 2005.

[11] J. E. Mark, Polymer Data Handbook, Oxford University Press, New York, NY, USA, 1999.

[12] J. Kuncová-Kallio and P. J. Kallio, "PDMS and its suitability for analytical microfluidic devices," in Proceedings of the 28th Annual International Conference of the IEEE Engineering in Medicine and Biology Society (EMBS '06), pp. 2486-2489, September 2006.

[13] H. S. El-Zaim and J. P. Heggers, "Silicones for pharmaceutical and biomedical applications," in Polymeric Biomaterials, Revised and Expanded, pp. 79-88, Marcel Dekker, New York, NY, USA, 2001.

[14] A. Mata, A. J. Fleischman, and S. Roy, "Characterization of polydimethylsiloxane (PDMS) properties for biomedical micro/nanosystems.", Biomedical Microdevices, vol. 7, no. 4, pp. 281-293, 2005.

[15] A. J. Moulson and J. M. Herbert, Electroceramics: Materials, Properties, Applications, John Wiley \& Sons, 2003.

[16] L. Guo, H. Luo, J. Gao, L. Guo, and J. Yang, "Microwave hydrothermal synthesis of barium titanate powders," Materials Letters, vol. 60, no. 24, pp. 3011-3014, 2006.

[17] H. Saito, H. Chazono, H. Kishi, and N. Yamaoka, "X7R multilayer ceramic capacitors with nickel electrodes," Japanese Journal of Applied Physics, vol. 30, no. 9, pp. 2307-2310, 1991.

[18] S. Chatterjee and H. S. Maiti, "Novel method of doping PTC thermistor sensor elements during sintering through diffusion by vapour phase," Materials Chemistry and Physics, vol. 67, no. 1-3, pp. 294-297, 2001.

[19] B. Huybrechts, K. Ishizaki, and M. Takata, "The positive temperature coefficient of resistivity in barium titanate," Journal of Materials Science, vol. 30, no. 10, pp. 2463-2474, 1995.

[20] S. Koulouridis, G. Kiziltas, Y. Zhou, D. J. Hansford, and J. L. Volakis, "Polymer-ceramic composites for microwave applications: fabrication and performance assessment," IEEE Transactions on Microwave Theory and Techniques, vol. 54, no. 12, pp. 4202-4208, 2006.

[21] Dow Corning Corporation, "Sylgard 184, material properties," http://www.dowcorning.com/.

[22] Sachtleben Pigments, Pori, Finland, http://www.sachtleben .com/.

[23] Ansoft HFSS, http://www.ansoft.com/products/hf/hfss/.
[24] Alien Technology. Higgs 2 product overview, http://www .alientechnology.com/docs/products/DS_H2.pdf.

[25] G. Marrocco, "The art of UHF RFID antenna design: impedance-matching and size-reduction techniques," IEEE Antennas and Propagation Magazine, vol. 50, no. 1, pp. 66-79, 2008.

[26] Agilent Technologies, "85070E Dielectric Probe Kit," http:// www.agilent.com/.

[27] S. Sankaralingam and B. Gupta, "Determination of dielectric constant of fabric materials and their use as substrates for design and development of antennas for wearable applications," IEEE Transactions on Instrumentation and Measurement, vol. 59, no. 12, pp. 3122-3130, 2010.

[28] K. Kurokawa, "Power waves and the scattering matrix," IEEE Transactions on Microwave Theory and Techniques, vol. 13, no. 2, pp. 194-202, 1965.

[29] P. V. Nikitin, K. V. S. Rao, S. F. Lam, V. Pillai, R. Martinez, and H. Heinrich, "Power reflection coefficient analysis for complex impedances in RFID tag design," IEEE Transactions on Microwave Theory and Techniques, vol. 53, no. 9, pp. 2721-2725, 2005.

[30] Voyantic, "RFID measurements solutions," http://www.voyantic.com/.

[31] J. Virtanen, T. Björninen, L. Ukkonen, and L. Sydänheimo, "Passive UHF inkjet-printed narrow-line RFID tags," IEEE Antennas and Wireless Propagation Letters, vol. 9, pp. 440-443, 2010.

[32] EPC frequency regulations UHF. 

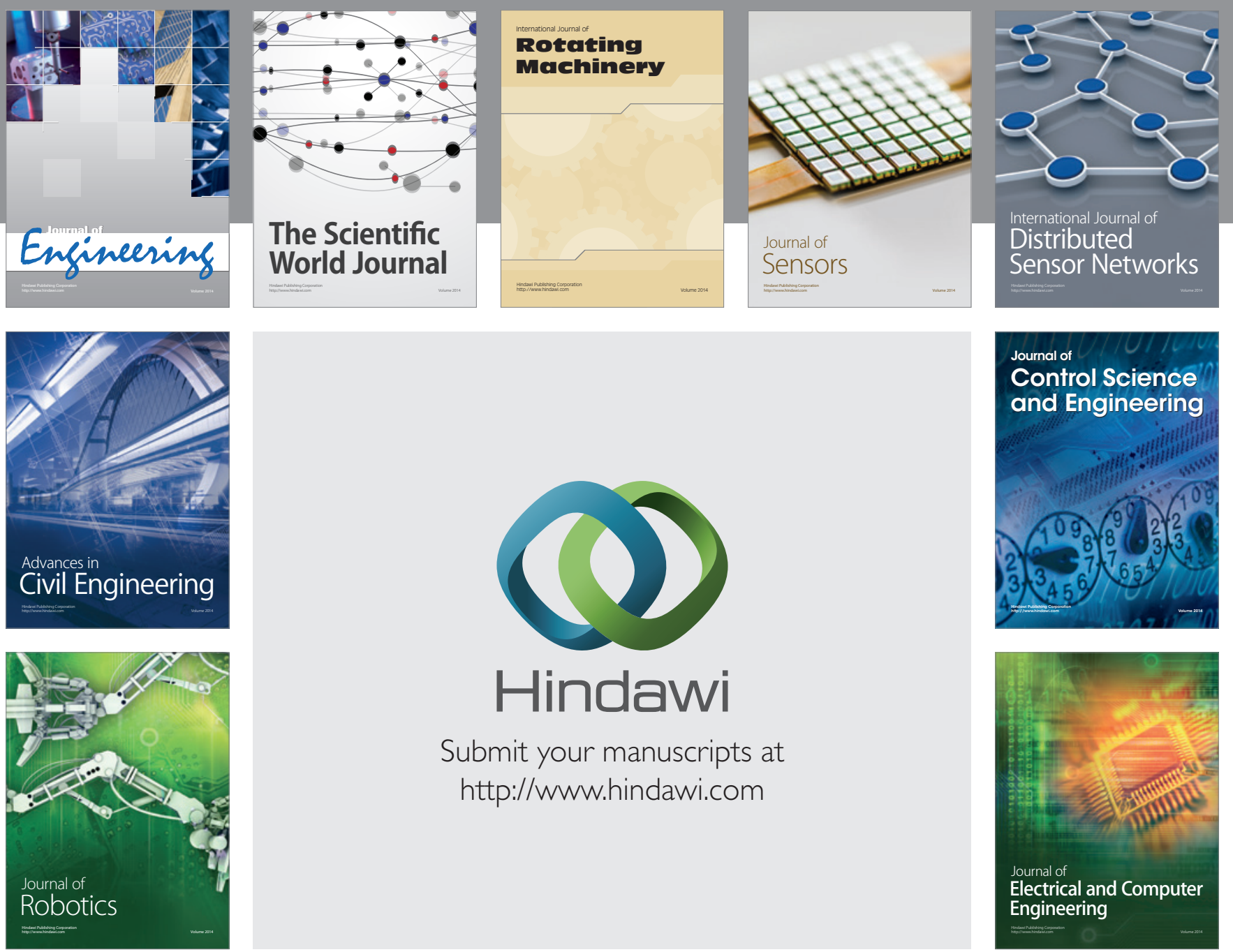

Submit your manuscripts at

http://www.hindawi.com
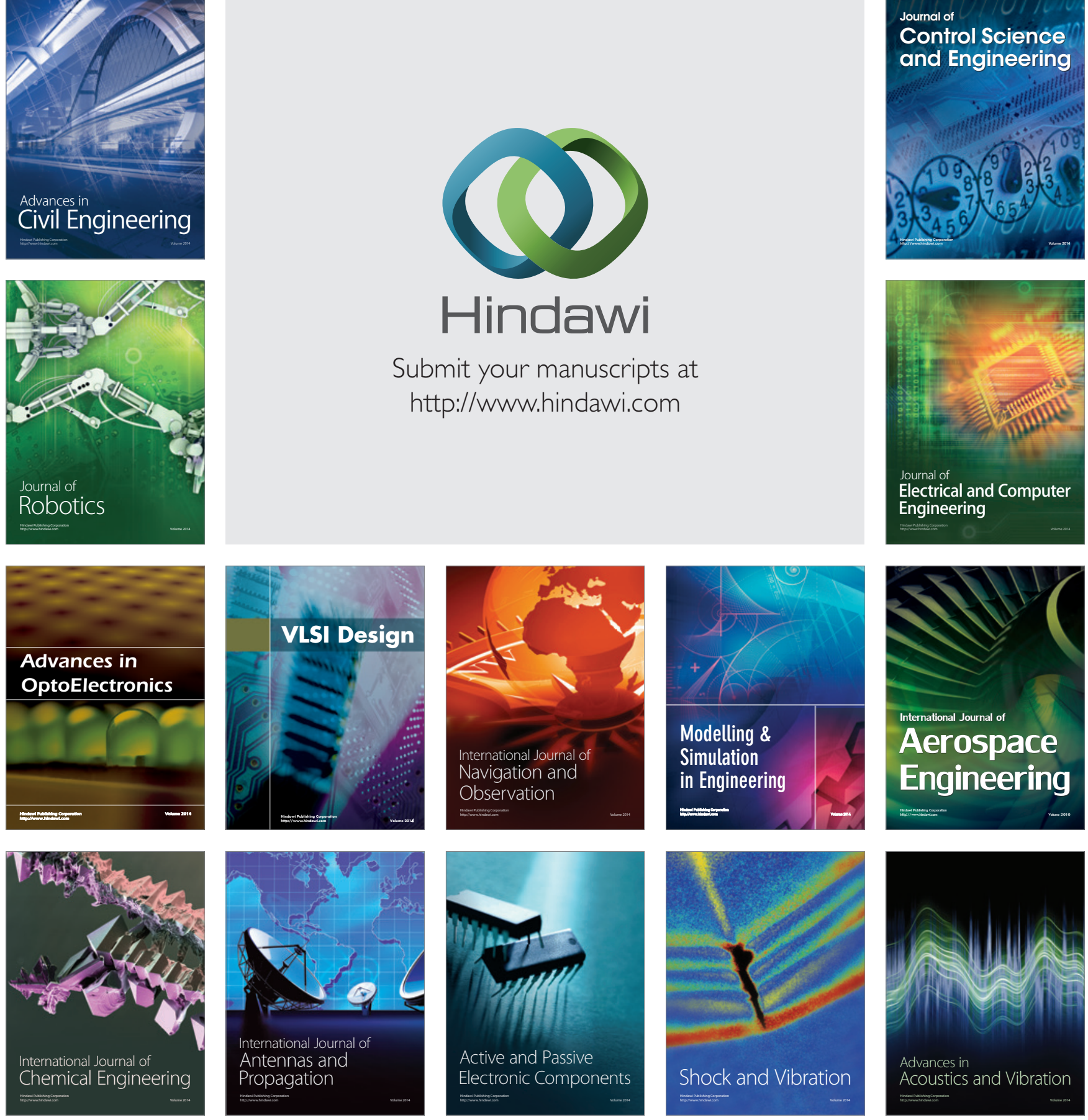